KYUNGPOOK Math. J. 55(2015), 127-135

http://dx.doi.org/10.5666/KMJ.2015.55.1.127

pISSN 1225-6951 eISSN 0454-8124

(C) Kyungpook Mathematical Journal

\title{
On Normalized Tight Frame Wavelet Sets
}

\author{
Swati SRIVAstava \\ Department of Mathematics, University of Allahabad, Allahabad, India \\ e-mail : swatisrivastava276@gmail.com
}

ABstract. We determine two-interval normalized tight frame wavelet sets for real dilation $d \in(1, \infty)$, and characterize all symmetric normalized tight frame wavelet sets. We also construct a normalized tight frame wavelet set which has an infinite number of components accumulating at the origin. These normalized tight frame wavelet sets and their closures possess the same measure. Finally an example of a normalized tight frame wavelet set is provided whose measure is strictly less than the measure of its closure.

\section{Introduction}

Wavelet sets introduced by Dai and Larson in 'Wandering vectors for unitary systems and wavelets, Mem. Amer. Math. Soc. 134 (1998)' have been put into the frame and tight frame setting by Han and Larson in [7]. For dilation $d \in(1, \infty)$, the orthonormal wavelets and the tight frames have been characterized by Chui and Shi in [3]. Wavelet sets having certain number of components have been studied by many workers in $[1,2,6,7,10$ and 11]. Characterizations of two-interval, three-interval and four-interval wavelet sets have been obtained for dilation $d$ using different methods.

After providing preliminaries in Section 2, we characterize two-interval frame wavelet sets for dilation $d$ in Section 3. Also, following the technique sponsored by Arcozzi, Behera and Madan to obtain $2 n$-interval symmetric wavelet sets for the dyadic dilation, we construct symmetric frame wavelet sets with real dilation $d$ in Section 4. In Section 5, we provide a frame wavelet set having infinite number of components that accumulates at the origin. Noting that the frame wavelet sets constructed in Section 4 and Section 5 have the measure equal to the measure of their respective closures, we provide a frame wavelet set in Section 6 which possesses a property that its measure is strictly less than that of its closure.

Received May 1, 2014; accepted July 17, 2014.

2010 Mathematics Subject Classification: 42C40, 65T60.

Key words and phrases: Wavelet, Frame, Wavelet set, Normalized tight frame wavelet set, Frame polygonal.

This work is supported by UGC India. 


\section{Preliminaries}

A sequence $\left\{x_{j}\right\}_{j \in \mathbb{J}}, \mathbb{J}$ being a countable index set, of vectors in a Hilbert space $\mathcal{H}$ is called a frame if there are constants $A, B>0$ such that

$$
A\|x\|^{2} \leq \sum_{j \in \mathbb{J}}\left|\left\langle x, x_{j}\right\rangle\right|^{2} \leq B\|x\|^{2}
$$

for all $x \in \mathcal{H}$, where $\langle\cdot, \cdot\rangle$ denotes the inner product on $\mathcal{H}$. The optimal constants are called the frame bounds. If the frame bounds are equal, the frame is called to be tight and if frame bounds equal 1 , it is called a normalized tight frame. A function $\psi \in L^{2}(\mathbb{R})$ is called a (tight, normalized tight) frame wavelet for dilation $d \in(1, \infty)$ if the system $\left\{\psi_{j, k}=d^{j / 2} \psi\left(d^{j} \cdot-k\right)\right\}_{j, k \in \mathbb{Z}}$ is a (tight, normalized tight) frame for $L^{2}(\mathbb{R})$. If the Fourier transform of a (tight, normalized tight) frame wavelet equals the characteristic function on a measurable set $E$, then the set $E$ is called a (tight, normalized tight) frame wavelet set. The Fourier transform of a function $f \in L^{1}(\mathbb{R}) \cap L^{2}(\mathbb{R})$ is defined for $\xi \in \mathbb{R}$ by

$$
\hat{f}(\xi)=\int_{\mathbb{R}} f(x) e^{-i x \xi} d x .
$$

Taking advantage of the fact that $L^{1}(\mathbb{R}) \cap L^{2}(\mathbb{R})$ is dense in $L^{2}(\mathbb{R})$, the definition of Fourier transform extends to the whole of $L^{2}(\mathbb{R})$.

In order to characterize a frame wavelet set, we provide the following well known definition [9].

Definition 2.1. Let $E$ and $F$ be two measurable subsets of $\mathbb{R}$. Then

(A) $E$ is said to be $2 \pi$-translation congruent to $F$, if there exists a measurable partition $\left\{E_{n}: n \in \mathbb{Z}\right\}$ of $E$ such that $\left\{E_{n}+2 n \pi: n \in \mathbb{Z}\right\}$ is a measurable partition of $F$.

(B) $E$ is said to be $d$-dilation congruent to $F$, if there exists a measurable partition $\left\{E_{n}: n \in \mathbb{Z}\right\}$ of $E$ such that $\left\{d^{n} E_{n}: n \in \mathbb{Z}\right\}$ is a measurable partition of $F$.

From [3, Corollary 4] and [5] we conclude that $\psi \in L^{2}(\mathbb{R})$, defined by $\hat{\psi}=\chi_{E}$ for a measurable set $E \subset \mathbb{R}$, provides a tight frame $\left\{\psi_{j, k}\right\}_{j, k \in \mathbb{Z}}$ with dilation $d \in(1, \infty)$, if and only if $E$ satisfies

$$
\sum_{k \in \mathbb{Z}} \chi_{E}(\xi+2 k \pi) \leq 1, \quad \xi \in \mathbb{R} ;
$$

and

$$
\sum_{j \in \mathbb{Z}} \chi_{E}\left(d^{j} \xi\right)=m, \quad \xi \in \mathbb{R}, \text { for some } m \in \mathbb{N} .
$$


Equivalently, a measurable set $E$ in $\mathbb{R}$ is a tight frame wavelet set if and only if the following hold:

(i) $^{\prime} E$ is $2 \pi$-translation congruent to a subset of $[c, c+2 \pi], c \in \mathbb{R}$;

and

(ii) $^{\prime} E$ is $d$-dilation congruent to $\left(-d^{m} a,-a\right) \cup\left(b, d^{m} b\right), a, b>0$ for some $m \in \mathbb{N}$.

It is pertinent to mention that a set in $\mathbb{R}$ which is both $2 \pi$-translation congruent and $d$-dilation congruent to a tight frame wavelet set is itself a tight frame wavelet set.

Following are some examples of tight frame wavelet sets.

Example 2.2. For $l, n, m \in \mathbb{N}$ and $k \geq 2$, the set $\left(-\frac{2 \pi}{k^{m}},-\frac{2 \pi}{k^{m+n}}\right) \cup\left(\frac{2 \pi}{k^{m+l}}, \frac{2 \pi}{k^{m}}\right)$ is a tight frame wavelet set for dilation $k$.

Example 2.3. For $k \geq 2$ and $A, B \subseteq \mathbb{N}$, the set $\bigcup_{\substack{n \in A \\ m \in B}}\left(\left(-\frac{2 \pi}{k^{m}},-\frac{2 \pi}{k^{m+1}}\right) \cup\right.$ $\left.\left(\frac{2 \pi}{k^{n+1}}, \frac{2 \pi}{k^{n}}\right)\right)$ is a tight frame wavelet set for dilation $k$.

Now onwards, we call a normalized tight frame wavelet set just a frame wavelet set. Hence, for a frame wavelet set the value of $m$ in (ii), and equivalently in (ii)', is 1. It is also to be mentioned that all equalities in this paper hold in the sense of almost everywhere.

\section{A Characterization of Two-interval Frame Wavelet Sets with Real Dilation $d$}

In this Section, we provide a characterization of frame wavelet sets having two intervals.

A measurable set $E \subset \mathbb{R}$ having two intervals must be of the form $(-d a,-a) \cup$ $(b, d b)$ to partition $\mathbb{R}$ by dilation $d$, for $a, b>0$. Also, for $E$ to be translation congruent to a subset of $[c, c+2 \pi], c \in \mathbb{R}$, we must have

$$
-a+2 n \pi \leq b \text { and }-d a+2(n+1) \pi \geq d b
$$

for some $n \in \mathbb{N}$, i.e.,

$$
a+b \geq 2 n \pi \text { and } d(a+b) \leq 2(n+1) \pi
$$

for some $n \in \mathbb{N}$.

Therefore, we have the following theorem.

Theorem 3.1. A measurable set $E$ in $\mathbb{R}$ is a frame wavelet set having two intervals with dilation d if and only if $E=(-d a,-a) \cup(b, d b)$ for $a, b>0$ satisfying (3.1).

Following are some examples of frame wavelet sets having two intervals. 
Example 3.2. For $n, m \in \mathbb{N}$ and $k \geq 2$, the set $\left(-\frac{2 \pi}{k^{m}},-\frac{2 \pi}{k^{m+1}}\right) \cup\left(\frac{2 \pi}{k^{n+1}}, \frac{2 \pi}{k^{n}}\right)$ is a frame wavelet set for $d=k$. In particular, the set $\left(-\frac{2 \pi}{2^{m}},-\frac{2 \pi}{2^{m+1}}\right) \cup\left(\frac{2 \pi}{2^{n+1}}, \frac{2 \pi}{2^{n}}\right)$ is a dyadic frame wavelet set for $n, m \in \mathbb{N}$.

Example 3.3. For $d=\frac{5}{3}$, the set $\left[-\frac{10}{3} \pi,-2 \pi\right] \cup\left[\frac{2}{5} \pi, \frac{2}{3} \pi\right]$ is a frame wavelet set.

Example 3.4. The set $[-4 \pi,-3 \pi] \cup[3 \pi, 4 \pi]$ is a frame wavelet set with dilation $d=\frac{4}{3}$, which is a wavelet set for the same dilation.

\section{A Construction of Symmetric Frame Wavelet Sets with Real Dilation $d$}

Following the method described in [1] to construct symmetric dyadic wavelet sets based on MSF polygonals we construct some frame wavelet sets. In addition, a characterization of all symmetric frame wavelet sets with real dilation $d>1$ has been provided.

In order to remain as self contained as possible, we enumerate following symbols and terminologies.

Consider the set $D$ of all points $P_{j}=\left(d^{\lambda_{j}}, d^{\lambda_{j}} m_{j}\right)$ in the euclidean plane for $m_{j} \in \mathbb{N}_{0}=\mathbb{N} \cup\{0\}$, and $\lambda_{j} \in \mathbb{Z}$. For a finite set $\mathcal{P}=\left\{P_{1}, P_{2}, \ldots, P_{n}\right\}$ in $D$,

$$
a^{j}=-\frac{d^{\lambda_{j}} m_{j}-d^{\lambda_{j+1}} m_{j+1}}{d^{\lambda_{j}}-d^{\lambda_{j+1}}}
$$

denotes the negative of the slope of the line joining $P_{j}$ and $P_{j+1}$.

We call the set $\mathcal{P}$ a frame polygonal if

$$
\lambda_{1}=0, \quad 0=a_{0}<a_{1}<\ldots<a_{n} \leq \frac{1}{2}
$$

and

$$
d^{\lambda_{n}}\left(m_{n}+a_{n}\right)=d m_{1}
$$

In [1], for $d=2$ and $a_{n}=\frac{1}{2}$, the frame polygonal is called the MSF polygonal.

Theorem 4.1. For a frame polygonal $\mathcal{P}$, let

$$
I_{j}=\left(\left[a_{j-1}, a_{j}\right]+m_{j}\right) \cdot 2 \pi \quad j=1,2, \ldots, n .
$$

Then $K=K^{+} \cup K^{-}$with $K^{+}=\bigcup_{j=1}^{n} I_{j}$ and $K^{-}=-K^{+}$is a symmetric frame wavelet set.

Proof. Due to (4.2) and the way $I_{j}$ 's are defined, $K^{+}$is $2 \pi$-translation congruent to a subset of $[0, \pi]$. Thus, by the symmetry, $K$ is $2 \pi$-translation congruent to a 
subset of $[-\pi, \pi]$.

Next, let $H_{j}=d^{\lambda_{j}} I_{j}, j=1,2, \ldots, n$. Using (4.1) and (4.3) one can show that $\bigcup_{j=1}^{n} H_{j}$ has the form $[\alpha, d \alpha]$ for some $\alpha>0$. Therefore, $K^{+}$is $d$-dilation congruent to $[\alpha, d \alpha]$. By the symmetry again, $K$ is $d$-dilation congruent to $[-d \alpha,-\alpha] \cup[\alpha, d \alpha]$ for some $\alpha>0$. Hence, $K$ is a frame wavelet set.

Now we illustrate the construction by means of some examples.

Example 4.2. For $n=3, d=4$ and $a_{3}=\frac{1}{4}$, let $P_{1}=\left(1,4^{s}(4 t+1)\right), P_{2}=$ $\left(4^{v}, 0\right), P_{3}=\left(4^{s+2}, 4^{s+2} t\right)$, where $s, t, v \in \mathbb{N}_{0}$ with $t \geq 1$ and $4^{v}>4^{s+2}(4 t+1)$. Then $\mathcal{P}=\left\{P_{1}, P_{2}, P_{3}\right\}$ is a frame polygonal with $a_{1}=\frac{4^{s}(4 t+1)}{4^{v}-1}, a_{2}=\frac{4^{s+2} t}{4^{v}-4^{s+2}}, a_{3}=\frac{1}{4}$. Hence

$$
\begin{gathered}
I_{1}=\left(\left[4^{s}(4 t+1), \frac{4^{s+v}(4 t+1)}{4^{v}-1}\right]\right) \cdot 2 \pi \\
I_{2}=\left(\left[\frac{4^{s}(4 t+1)}{4^{v}-1}, \frac{4^{s+2} t}{4^{v}-4^{s+2}}\right]\right) \cdot 2 \pi \\
I_{3}=\left(\left[\frac{4^{v} t}{4^{v}-4^{s+2}}, \frac{4 t+1}{4}\right]\right) \cdot 2 \pi .
\end{gathered}
$$

Therefore, with $K^{+}=\bigcup_{i=1}^{3} I_{i}$ and $K^{-}=-K^{+}, K=K^{+} \cup K^{-}$is a frame wavelet set.

Example 4.3. For $n=3, d=2$ and $a_{3}=\frac{1}{2^{p}}, p \in \mathbb{N}$, the frame wavelet set is found to be

$$
\begin{gathered}
\pm\left(\left[2^{s}\left(2^{p} t+1\right), \frac{2^{s+v}\left(2^{p} t+1\right)}{2^{v}-1}\right] \cup\left[\frac{2^{s}\left(2^{p} t+1\right)}{2^{v}-1}, \frac{2^{s+p+1} t}{2^{v}-2^{s+p+1}}\right]\right. \\
\left.\cup\left[\frac{2^{v} t}{2^{v}-2^{s+p+1}}, \frac{2^{p} t+1}{2^{p}}\right]\right) \cdot 2 \pi
\end{gathered}
$$

on taking $P_{1}=\left(1,2^{s}\left(2^{p} t+1\right)\right), P_{2}=\left(2^{v}, 0\right), P_{3}=\left(2^{s+p+1}, 2^{s+p+1} t\right)$, where $s, t, v \in$ $\mathbb{N}_{0}$ with $t \geq 1$ and $2^{v}>2^{s+p+1}\left(2^{p} t+1\right)$.

Example 4.4. For $n=2, d=2$ and $a_{3}=\frac{1}{2^{p}}, p \in \mathbb{N}$, let $P_{1}=\left(1,2^{l}(2 t+1)\right), l, t \in$ $\mathbb{N}_{0}$. Using (4.3) we can find $P_{2}=\left(2^{l+p+1}, 2^{l+2} t\right)$. Then $\left\{P_{1}, P_{2}\right\}$ forms a frame polygonal if

$$
a_{1}=\frac{2^{l}-2^{l+1} t}{2^{l+p+1}-1}>0,
$$

which shows that $t$ must be 0 and hence we get the frame wavelet set $K=K^{+} \cup K^{-}$, where

$$
K^{+}=\left(\left[\frac{2^{l}}{2^{l+p+1}-1}, \frac{1}{2^{p}}\right] \cup\left[2^{l}, \frac{2^{2 l+p+1}}{2^{l+p+1}-1}\right]\right) \cdot 2 \pi
$$


and $K^{-}=-K^{+}$.

In the following theorem we characterize all symmetric frame wavelet sets with real dilation $d>1$.

Theorem 4.5. A measurable set $E \subset \mathbb{R}$ is a symmetric frame wavelet set with dilation $d>1$ if and only if $E=E^{+} \cup E^{-}$with $E^{+}=\bigcup_{\alpha \in A} I_{\alpha}$, and $E^{-}=-E^{+}$, where

(a) $A \subseteq\{1,2, \ldots, n\}$,

(b) for $j=1,2, \ldots, n, I_{j}=\left(\epsilon_{j}\left[a_{j-1}, a_{j}\right]+m_{j}\right) \cdot 2 \pi$ with $\epsilon_{j} \in\{-1,1\}, m_{j} \in \mathbb{N}_{0}$ and $a_{j}$ 's satisfying (4.2), and,

(c) $\bigcup_{\alpha \in A} H_{\alpha}$, where $H_{\alpha}=d^{\lambda_{\tau(\alpha)}} I_{\tau(\alpha)}, \alpha \in A, \lambda_{j} \in \mathbb{Z}, \tau$ is a permutation on $A$, has the form $[b, d b]$ for some $b>0$.

Proof. The definition of $I_{j}$ 's is equivalent to the condition that $E$ is $2 \pi$-translation congruent to a subset of $[-\pi, \pi]$. Also, the definition and the condition on $H_{\alpha}$ 's is equivalent to the condition for $E$ to be $d$-dilation congruent to $[-d b,-b] \cup[b, d b]$.

Note that the frame wavelet sets constructed in this Section have finite number of components. In the next Section we construct a frame wavelet set which has an infinite number of components and accumulates at the origin.

\section{A Construction of a Frame Wavelet Set Accumulating at the Origin}

For $n, p \in \mathbb{N}, n \geq 2$, let $K^{+}=\left(K_{1} \cup K_{2}\right) \cdot 2 \pi$, where

$$
K_{1}=\left[\frac{2^{n-2}}{2^{n+p-1}-1}, \frac{1}{2^{p}}\right]
$$

and

$$
K_{2}=\left[2^{n-2}, \frac{2^{2 n+p-3}}{2^{n+p-1}-1}\right]
$$

and $K^{-}=-K^{+}$. Then $K=K^{+} \cup K^{-}$is a frame wavelet set by Example 4.4.

Put $a_{n}=\frac{2^{n-2}}{2^{n+p-1}-1}$. Thus $K_{1}=\left[a_{n}, \frac{1}{2^{p}}\right]$ and $K_{2}=\left[2^{n-2}, 2^{n+p-1} a_{n}\right]$. For $0<\epsilon<\frac{1}{2^{p+1}}\left(\frac{2^{n+p-2}-1}{2^{n+p-1}-1}\right)$, construct

$$
\begin{gathered}
S_{1}=\left[\frac{a_{n}}{2}+\frac{\epsilon}{2^{n+p-1}}, \frac{a_{n}}{2}+\epsilon\right], \\
S_{2}=\left[a_{n}+2 \epsilon, \frac{1}{2^{p}}\right]
\end{gathered}
$$


and

$$
S_{3}=\left[2^{n+p-1} a_{n}, 2^{n+p-1} a_{n}+2 \epsilon\right]
$$

and set

$$
\begin{gathered}
E_{0}=S_{1}+2^{n-2}, \quad F_{0}=2^{-(n+p)} E_{0}, \\
E_{l}=F_{l-1}+2^{n-2}, \quad F_{l}=2^{-(n+p+l)} E_{l}, \quad l \in \mathbb{N} .
\end{gathered}
$$

Let

$$
F^{+}=\left[\left(K_{2}-\bigcup_{l=0}^{\infty} E_{l}\right) \cup \bigcup_{l=0}^{\infty} F_{l} \cup S_{1} \cup S_{2} \cup S_{3}\right] \cdot 2 \pi .
$$

Noting that $E_{l} \subset K_{2}$ for all $l \in \mathbb{N}_{0}$, we can see that

$$
\frac{1}{2^{n+p-1}} S_{3} \cup 2 S_{1} \cup S_{2}=K_{1}
$$

and

$$
\left(K_{2}-\bigcup_{l=0}^{\infty} E_{l}\right) \cup\left(\bigcup_{l=0}^{\infty} 2^{n+p+l} F_{l}\right)=K_{2}
$$

Thus $F^{+}$is 2-dilation congruent to $K^{+}$. Also, we have

$$
S_{2} \cup\left(S_{3}-2^{n-2}\right)=K_{1}
$$

and

$$
\left(K_{2}-\bigcup_{l=0}^{\infty} E_{l}\right) \cup\left(\bigcup_{l=0}^{\infty}\left(F_{l}+2^{n-2}\right)\right) \cup\left(S_{1}+2^{n-2}\right)=K_{2} .
$$

Thus $F^{+}$is $2 \pi$-translation congruent to $K^{+}$.

Let $F^{-}=-F^{+}$and $F=F^{+} \cup F^{-}$. Then, by the symmetry, $F$ is both 2-dilation congruent and $2 \pi$-translation congruent to $K$. Hence $F$ is a frame wavelet set. Thus we conclude the following theorem.

Theorem 5.1. The set $F=F^{+} \cup F^{-}$, where $F^{+}$is given by (5.1) and $F^{-}=-F^{+}$, is a frame wavelet set that accumulates at the origin.

It is clear that $F$ contains an infinite number of components and accumulates at the origin. Also, it can be noticed here that $F$ and the closure $\bar{F}$ of $F$ have the same measure which is equal to that of $K$. In the next Section we provide a frame wavelet set whose measure is strictly less than that of its closure.

\section{A Construction of a Frame Wavelet Set whose Measure is Strictly Less than the Measure of its Closure}

In [12], it is shown that there is a wavelet set $E$ such that its closure $\bar{E}$ satisfies 
$|\bar{E}|>|E|$, where $|E|$ represents the measure of the set $E$. We show that the same is true for a frame wavelet set. Precisely, we construct a frame wavelet set for the dyadic dilation whose measure is strictly less than the measure of its closure.

Consider a countable dense set $\left\{\omega_{n}\right\}_{n=1}^{\infty}$ in the interval $\left[\frac{\pi}{3}, \frac{2 \pi}{3}\right]$.

Let

$$
\begin{gathered}
F_{1}=\bigcup_{n=1}^{\infty}\left(\left(\omega_{n}-\frac{\pi}{2^{n+3}}, \omega_{n}+\frac{\pi}{2^{n+3}}\right) \cap\left[\frac{\pi}{3}, \frac{2 \pi}{3}\right]\right), \\
F_{2}=\left[\frac{2 \pi}{3}, \frac{4 \pi}{3}\right]-2 F_{1} \text { and } F_{3}=\left[-\frac{2 \pi}{3},-\frac{\pi}{3}\right] .
\end{gathered}
$$

Set

$$
K=\bigcup_{n=1}^{3} F_{i}
$$

We claim that $K$ is a frame wavelet set. For, $2 F_{1} \cup F_{2}=\left[\frac{2 \pi}{3}, \frac{4 \pi}{3}\right]$ and $F_{3}=$ $\left[-\frac{2 \pi}{3},-\frac{\pi}{3}\right]$, thus $K$ is 2-dilation congruent to the set $(-2 \alpha,-\alpha) \cup(\beta, 2 \beta)$ for $\alpha=\frac{\pi}{3}$ and $\beta=\frac{2 \pi}{3}$. Also, $F_{1} \subset\left[\frac{\pi}{3}, \frac{2 \pi}{3}\right], F_{2} \subset\left[\frac{2 \pi}{3}, \frac{4 \pi}{3}\right], F_{3}+2 \pi \subset\left[\frac{4 \pi}{3}, \frac{5 \pi}{3}\right]$ and hence $K$ is $2 \pi$-translation congruent to a subset of $\left[\frac{\pi}{3}, \frac{5 \pi}{3}\right] \subset\left[\frac{\pi}{3}, \frac{\pi}{3}+2 \pi\right]$. Thus $K$ is a frame wavelet set for the dyadic dilation.

Next we show that $|\bar{K}|>|K|$. Since $\left\{\omega_{n}\right\}_{n=1}^{\infty}$ is dense in $\left[\frac{\pi}{3}, \frac{2 \pi}{3}\right]$, we have $\bar{F}_{1}=\left[\frac{\pi}{3}, \frac{2 \pi}{3}\right]$ and hence $\left|\bar{F}_{1}\right|=\frac{\pi}{3}$, while $\left|F_{1}\right| \leq \frac{\pi}{4}$. Thus $\left|F_{1}\right|<\left|\bar{F}_{1}\right|$. Also, $\left|F_{i}\right| \leq\left|\bar{F}_{i}\right|$ for $i=2,3$. Therefore

$$
|K|=\left|\bigcup_{n=1}^{3} F_{i}\right|=\sum_{n=1}^{3}\left|F_{i}\right|<\sum_{n=1}^{3}\left|\bar{F}_{i}\right|=|\bar{K}| .
$$

Hence $K$ is a frame wavelet set whose measure is strictly less than the measure of its closure. Thus we have,

Theorem 6.1. There exists a frame wavelet set $K$ such that $|K|$ is strictly less than $|\bar{K}|$.

\section{References}

[1] N. Arcozzi, B. Behera and S. Madan, Large classes of minimally supported frequency wavelets of $L^{2}(\mathbb{R})$ and $H^{2}(\mathbb{R})$, J. Geom. Anal., 13(2003), 557-579.

[2] M. Bownik and K. R. Hoover, Dimension functions of rationally dilated GMRA's and wavelets, J. Fourier Anal. Appl. 15 (2009), 585-615. 
[3] C. K. Chui and X. Shi, Orthonormal wavelets and tight frames with arbitrary real dilations, Appl. Comput. Harmon. Anal., 9(2000), 243-264.

[4] O. Cristensen, An Introduction to Frames and Reisz Bases, Birkhäuser, 2003.

[5] X. Dai, Y. Diao and Q. Gu, Frame wavelet sets in $\mathbb{R}$, Proc. Amer. Math. Soc., 132(2004), 2567-2575.

[6] Y.-H. Ha, H. Kang, J. Lee and J. K. Seo, Unimodular wavelets for $L^{2}$ and the Hardy space $H^{2}$, Michigan Math. J., 41(1994), 345-361.

[7] D. Han and D. R. Larson, Frames, bases and group representation, Mem. Amer. Math. Soc. $697,2000$.

[8] E. Hernández and G. Weiss, A First Course on Wavelets, CRC Press, 1996.

[9] D. R. Larson, Unitary systems and wavelet sets, Wavelet Analysis and Applications, Birkhäuser Basel, 2007, 143-171.

[10] D. Singh, On Multiresolution Analysis, D. Phil. Thesis, University of Allahabad, 2010.

[11] N. K. Shukla and G. C. S. Yadav, A characterization of three-interval scaling sets, Real Anal. Exchange, 35(2009), 121-138.

[12] Z. Zhang, The measure of the closure of a wavelet set may be $>2 \pi$, J. Cohen and A. I. Zayed (eds.), Wavelets and Multiscale Analysis: Theory and Applications, Applied and Numerical Harmonic Analysis, Springer, 2011. 